University of Wollongong

Research Online

Faculty of Social Sciences - Papers (Archive) Faculty of Arts, Social Sciences \& Humanities

$1-1-2018$

Does Partner Selection Mediate the Relationship Between School Attendance and HIV/Herpes Simplex Virus-2 Among Adolescent Girls and Young Women in South Africa: An Analysis of HIV Prevention Trials Network 068 Data

\author{
Marie Stoner \\ University of North Carolina \\ Jessie Edwards \\ University of North Carolina \\ William Miller \\ The Ohio State University \\ Allison Aiello \\ University of North Carolina \\ Carolyn Halpern \\ University of North Carolina
}

See next page for additional authors

Follow this and additional works at: https://ro.uow.edu.au/sspapers

Part of the Education Commons, and the Social and Behavioral Sciences Commons

Research Online is the open access institutional repository for the University of Wollongong. For further information contact the UOW Library: research-pubs@uow.edu.au 


\title{
Does Partner Selection Mediate the Relationship Between School Attendance and HIV/Herpes Simplex Virus-2 Among Adolescent Girls and Young Women in South Africa: An Analysis of HIV Prevention Trials Network 068 Data
}

\author{
Abstract \\ Objective: School attendance prevents HIV and herpes simplex virus-2 (HSV-2) in adolescent girls and \\ young women, but the mechanisms to explain this relationship remain unclear. Our study assesses the \\ extent to which characteristics of sex partners, partner age, and number mediate the relationship \\ between attendance and risk of infection in adolescent girls and young women in South Africa.
}

Design: We use longitudinal data from the HIV Prevention Trials Network 068 randomized controlled trial in rural South Africa, where girls were enrolled in early adolescence and followed in the main trial for more than 3 years. We examined older partners and the number of partners as possible mediators.

Methods: We used the parametric g-formula to estimate 4-year risk differences for the effect of school attendance on the cumulative incidence of HIV/HSV-2 overall and the controlled direct effect (CDE) for mediation. We examined mediation separately and jointly for the mediators of interest.

Results: We found that young women with high attendance in school had a lower cumulative incidence of HIV compared with those with low attendance (risk difference $=-1.6 \%)$. Partner age difference $(\mathrm{CDE}=$ $-1.2 \%)$ and the number of partners (CDE $=-0.4 \%$ ) mediated a large portion of this effect. In fact, when we accounted for the mediators jointly, the effect of schooling on HIV was almost removed, showing full mediation $(\mathrm{CDE}=-0.3 \%)$. The same patterns were observed for the relationship between school attendance and cumulative incidence of HSV-2 infection.

Conclusion: Increasing school attendance reduces the risk of acquiring HIV and HSV-2. Our results indicate the importance of school attendance in reducing partner number and partner age difference in this relationship.

Disciplines

Education | Social and Behavioral Sciences

Publication Details

Stoner, M. C. D., Edwards, J. K., Miller, W. C., Aiello, A. E., Halpern, C. T., Julien, A., Rucinski, K. B., Selin, A., Twine, R., Hughes, J. P., Wang, J., Agyei, Y., Xavier Gomez-Olive, F., Wagner, R. G., Laeyendecker, O., MacPhail, C., Kahn, K. \& Pettifor, A. (2018). Does Partner Selection Mediate the Relationship Between School Attendance and HIV/Herpes Simplex Virus-2 Among Adolescent Girls and Young Women in South Africa: An Analysis of HIV Prevention Trials Network 068 Data. JAIDS: Journal of Acquired Immune Deficiency Syndromes, 79 (1), 20-27.

\section{Authors}

Marie Stoner, Jessie Edwards, William Miller, Allison Aiello, Carolyn Halpern, Aimee Julien, Katherine B. Rucinski, Amanda Selin, Rhian Twine, James Hughes, Jing Wang, Yaw Agyei, Francesc Xavier GomezOlive, Ryan Wagner, Oliver Laeyendecker, Catherine L. Mac Phail, Kathleen Kahn, and Audrey Pettifor 


\title{
Does Partner Selection Mediate the Relationship Between School Attendance and HIV/Herpes Simplex Virus-2 Among Adolescent Girls and Young Women in South Africa: An Analysis of HIV Prevention Trials Network 068 Data
}

\author{
Marie C. D. Stoner, PhD, * Jessie K. Edwards, PhD, * William C. Miller, PhD, † Allison E. Aiello, PhD,* \\ Carolyn T. Halpern, PhD, $\neq$ Aimée Julien, MPH, ${ }^{*}$ Katherine B. Rucinski, MPH, * Amanda Selin, MHS,* \\ Rhian Twine, MPH, § James P. Hughes, PhD,\|ף Jing Wang, MS, \ Yaw Agyei, MPH,\#

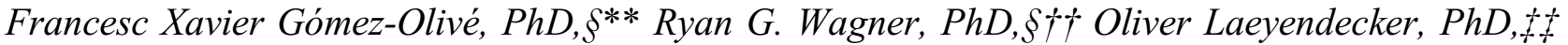 \\ Catherine Macphail, PhD, $\S \xi\|\|$ Kathleen Kahn, PhD, $\S^{* *}+\dagger$ and Audrey Pettifor, PhD* $\S$
}

\begin{abstract}
Objective: School attendance prevents HIV and herpes simplex virus-2 (HSV-2) in adolescent girls and young women, but the mechanisms to explain this relationship remain unclear. Our study assesses the extent to which characteristics of sex partners, partner age, and number mediate the relationship between attendance and risk of infection in adolescent girls and young women in South Africa.
\end{abstract}

Design: We use longitudinal data from the HIV Prevention Trials Network 068 randomized controlled trial in rural South Africa, where girls were enrolled in early adolescence and followed in the main trial for more than 3 years. We examined older partners and the number of partners as possible mediators.

Methods: We used the parametric g-formula to estimate 4-year risk differences for the effect of school attendance on the cumulative incidence of HIV/HSV-2 overall and the controlled direct effect (CDE) for mediation. We examined mediation separately and jointly for the mediators of interest.

Results: We found that young women with high attendance in school had a lower cumulative incidence of HIV compared with those with low attendance (risk difference $=-1.6 \%$ ). Partner age
Received for publication March 2, 2018; accepted May 9, 2018

From the *Department of Epidemiology, University of North Carolina, Chapel Hill, NC; †Division of Epidemiology, The Ohio State University, Columbus, $\mathrm{OH} ; \vdots$ Department of Maternal and Child Health, University of North Carolina, Chapel Hill, NC; §MRC/Wits Rural Public Health and Health Transitions Research Unit (Agincourt), School of Public Health, Faculty of Health Sciences, University of the Witwatersrand, Johannesburg, South Africa; \|Department of Biostatistics, University of Washington, Seattle, WA; -Fred Hutchinson Cancer Research Center, Seattle, WA; \#School of Medicine, Johns Hopkins University, Baltimore, MD; **INDEPTH Network, Accra, Ghana; ††Department of Public Health and Clinical Medicine, Epidemiology and Global Health Unit, Umeå University, Umeå, Sweden; \$\$Division of Intramural Research, National Institute of Allergy and Infectious Diseases, National Institutes of Health, Baltimore, MD; $\S \S S c h o o l$ of Health and Society, University of Wollongong, Wollongong, New South Wales, Australia; and \|\| Wits Reproductive Health and HIV Research Institute, University of the Witwatersrand, Johannesburg, South Africa.

Supported by T32 5T32AI007001, R01 MH110186 from the National Institutes of Health (NIH) and by Award Numbers UM1 AI068619 (HPTN Leadership and Operations Center), UM1AI068617 (HPTN Statistical and Data Management Center), and UM1AI068613 (HPTN Laboratory Center) from the National Institute of Allergy and Infectious Diseases, the National Institute of Mental Health, and the National Institute on Drug Abuse of the National Institutes of Health. This work was also supported by NIMH R01 (R01MH087118) and the Carolina Population Center and its NIH Center grant (P2C HD050924). The MRC/Wits Rural Public Health and Health Transitions Research Unit and Agincourt Health and Socio-Demographic Surveillance System have been supported by the University of the Witwatersrand, the Medical Research Council, South Africa, and the Wellcome Trust, UK (grants 058893/Z/99/A; 069683/Z/02/Z; 085477/Z/08/Z; $085477 / \mathrm{B} / 08 / \mathrm{Z})$. The content is solely the responsibility of the authors and does not necessarily represent the official views of the National Institutes of Health.

Planning to submit to AIDS 2018 Conference; Amsterdam, the Netherlands; July $23-27$.

The authors have no funding or conflicts of interest to disclose.

Similar prior publications by the first author using the same data source include the following: (1) M.C.D.S., J.K.E., W.C.M., A.E.A, C.T.H., A.S., J.P.H., J.W., O.L., Y.A., McPhail C, K.K., A.P. (2017). The effect of schooling on incident HIV and HSV-2 infection in young South African women enrolled in HPTN 068. AIDS. 24; 31:2127-2213. PMCID: PMC5599334. (2) M.C.D.S., J.K.E., W.C.M., A.E.A, C.T.H., Julien Suarez, A.S., J.P.H., J.W., McPhail C, K.K., A.P. (2017). The effect of schooling on age-disparate relationships and number of sexual partners among young women in rural South Africa enrolled in HPTN 068. J Acquir Immune Defic Syndr. 76:e107-e114. PMCID: PMC56801112.

A.P., J.K.E., A.E.A., K.B.R., W.C.M. and C.T.H. contributed to the conception, design of the analysis, and review of the writing. The remaining authors were involved in data acquisition, data collection, study management, and design of the original parent study. Additionally, J.K.E. and J.P.H. contributed to the analysis of data.

Supplemental digital content is available for this article. Direct URL citations appear in the printed text and are provided in the HTML and PDF versions of this article on the journal's Web site (www.jaids.com).

Correspondence to: Marie C. D. Stoner, PhD, Department of Epidemiology, University of North Carolina, Chapel Hill, NC 27599 (e-mail: stonerm@, email.unc.edu).

Copyright (C) 2018 The Author(s). Published by Wolters Kluwer Health, Inc. This is an open-access article distributed under the terms of the Creative Commons Attribution-Non Commercial License 4.0 (CCBY-NC), where it is permissible to download, share, remix, transform, and buildup the work provided it is properly cited. The work cannot be used commercially without permission from the journal. 
difference $(\mathrm{CDE}=-1.2 \%)$ and the number of partners $(\mathrm{CDE}=$ $-0.4 \%$ ) mediated a large portion of this effect. In fact, when we accounted for the mediators jointly, the effect of schooling on HIV was almost removed, showing full mediation ( $\mathrm{CDE}=-0.3 \%)$. The same patterns were observed for the relationship between school attendance and cumulative incidence of HSV-2 infection.

Conclusion: Increasing school attendance reduces the risk of acquiring HIV and HSV-2. Our results indicate the importance of school attendance in reducing partner number and partner age difference in this relationship.

Key Words: South Africa, adolescent girls and young women, HIV, HSV-2, education, mediation

(J Acquir Immune Defic Syndr 2018;79:20-27)

\section{INTRODUCTION}

Young South African women have an extremely high burden of HIV and herpes simplex v-II (HSV-2). The prevalence of HIV in adolescent girls and young women (AGYW) aged 15-26 years is $16 \%,{ }^{1}$ and the prevalence of HSV-2 is $29 \% .^{2}$ Most interventions to prevent sexually transmitted infections in AGYW have focused on modifying sexual risk behaviors and have had limited success. ${ }^{3-5}$ However, there is now a large body of evidence showing that attending school and staying in school protect against HIV and HSV-2 infection. ${ }^{6-10}$ Our analysis from South Africa showed that low school attendance and school dropout were associated with over twice the risk of both incident HIV and incident HSV-2. ${ }^{11}$ Yet, the mechanisms underlying the relationship between attending school and sexually transmitted infections are not understood.

HIV and HSV-2 are most commonly transmitted sexually. Therefore, for schooling to affect acquisition of HIV and HSV-2, schooling must influence behavioral factors that can affect the likelihood of transmission such as exposure to infection through a sexual partner. ${ }^{12}$ Researchers have hypothesized that the effect of education on HIV risk, including both educational attainment and school attendance, may be a result of changes in social networks, self-efficacy, socioeconomic status (SES), or sexual risk behaviors. However, there is limited empirical evidence investigating pathways between education and HIV or HSV2 infection. ${ }^{3}$ Because of associations between school attendance and both partner age difference and partner number and theories on the importance of sexual networks in individual acquisition of HIV, we chose to examine the mediating effect of characteristics of sex partners in the association between school attendance and HIV risk reduction. ${ }^{7,13-16}$

Our hypothesis is based on the idea that the structure of partnership networks is important in influencing young women's HIV risk. ${ }^{17,18}$ Social control theory and the routine/time use perspective state that behavior (in this case sexual behavior) can be limited by social forces and that activities that involve structured, supervised time will limit adolescent deviant or risky behavior. ${ }^{19-24}$ We theorized that the supervised, structured environment of school would lead to fewer partners as AGYW are occupied and to younger partners (who are less likely to have HIV), as students spend more time socializing with individuals in their peer group. Our previous study from South Africa showed that the girls who attend school have partners closer in age to themselves and fewer partners. ${ }^{16}$ Therefore, it is possible that AGYW in school are less likely to become infected with HIV and HSV-2 because school attendance shapes their sexual network by influencing the types of partners they choose. No studies have directly examined if sex partners mediate the relationship between school attendance and HIV acquisition.

Given that attending school is one of the few factors that is strongly preventative against HIV and HSV-2 infection for adolescent girls, understanding the pathways through which school reduces the risk is important to improve our

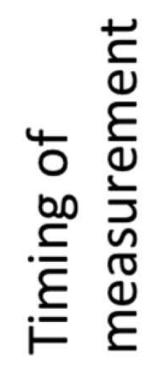

Exposure 3 Mediators 3 Outcome 3

Exposure 2 Mediators 2 Outcome 2

Exposure 1 Mediators 1 Outcome 1

FIGURE 1. Timing and measurement of exposure, outcome, and mediators in a participant with all possible visits.

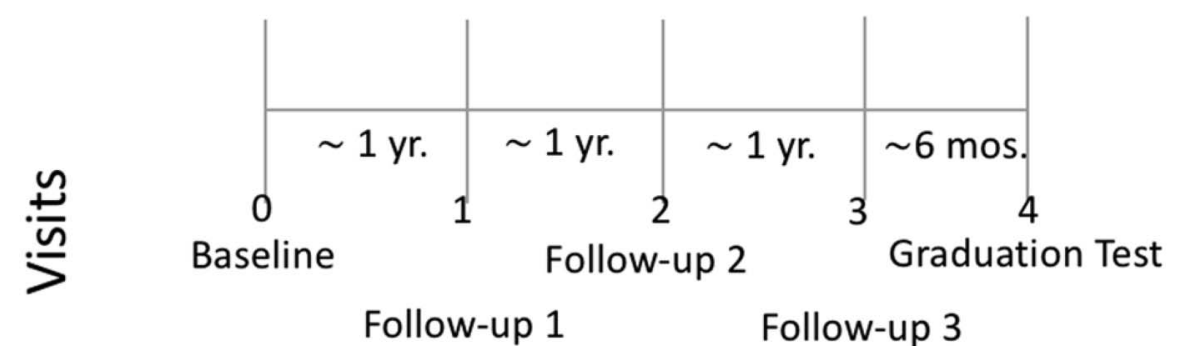


prevention response. Our study explores if partner age difference or the number of partners mediate the relationships between school attendance and incident HIV and HSV-2 infection among AGYW.

\section{METHODS}

\section{Study Population}

We used data from the HIV Prevention Trials Network (HPTN) 068 study, a phase III randomized trial to determine whether providing cash transfers, conditional on school attendance, reduced the risk of HIV acquisition in young women. ${ }^{25,26}$ Details of the behavioral questionnaire and laboratory data are available in the parent publication of the trial. ${ }^{26}$ The study included young women living in 28 villages within the MRC/ Wits Rural Public Health and Health Transitions Research Unit (Agincourt) in rural Mpumalanga Province, South Africa. The study enrolled 2533 young women aged 13-20 years in high school grades $8,9,10$, or 11 . Young women who were pregnant or married at enrollment or had no parent/guardian in the household were excluded. To assess incident HIV infection and mediation, we only included young women who had at least 2 follow-up visits and were HIV negative at enrollment and at the first follow-up visit (Fig. 1). We did so to ensure that school attendance was measured before the potential mediators, and the mediators were measured before the outcome. For incident

TABLE 1. Baseline Characteristics of Young Women Aged 13 to 20 Without Prevalent HIV Infection and at Least Follow-Up Visits in Agincourt, South Africa From March 2011 to December $2012(\mathrm{~N}=2086)^{*}$

\begin{tabular}{|c|c|}
\hline & n $(\%)$ \\
\hline \multicolumn{2}{|l|}{ Young women's age at baseline (yr) } \\
\hline $13-14$ & $717(34.4)$ \\
\hline $15-16$ & $913(43.8)$ \\
\hline $17-18$ & $384(18.4)$ \\
\hline $18-20$ & $72(3.5)$ \\
\hline \multicolumn{2}{|l|}{ Household wealth } \\
\hline Low & $540(25.9)$ \\
\hline Middle to low & $566(27.2)$ \\
\hline Middle & $489(23.5)$ \\
\hline High & $488(23.4)$ \\
\hline CCT randomization arm & $1091(52.3)$ \\
\hline Partner 5 or more years older & $105(5.1)$ \\
\hline Ever pregnant or had a child & $150(7.3)$ \\
\hline Prevalent HSV-2 infection & $73(3.5)$ \\
\hline Any alcohol use & $173(8.3)$ \\
\hline Double or single orphan & $1314(30.2)$ \\
\hline Children's depression inventory score $\geq 7$ & $369(17.7)$ \\
\hline Revised children's manifest anxiety score $\geq 7$ & $570(27.3)$ \\
\hline \multicolumn{2}{|l|}{ Partner number } \\
\hline 0 & $1626(78.9)$ \\
\hline 1 & $344(16.7)$ \\
\hline$\geq 2$ & $92(4.5)$ \\
\hline $\begin{array}{l}\text { *Missing data in observed: age, } 0 \text {; SES, } 3 \text {; age d } \\
\text { status, 2; alcohol, 3; orphan, 98; depression, } 0 \text {; anxie } \\
\text { CCT, Conditional Cash Transfer. }\end{array}$ & $\begin{array}{l}\text { ant, } 23 ; \mathrm{HSV}-2 \\
\text { oer, } \mathrm{n}=24\end{array}$ \\
\hline
\end{tabular}

HSV-2 infection, we further excluded prevalent cases of HSV-2 at enrollment or the first follow-up.

Young women were seen annually from baseline until study completion or expected graduation from high school. Each annual study visit included an audio computer-assisted selfinterview with the young woman and HIV and HSV-2 testing for those who were negative at the previous visit. Up to 4 assessments of the young women were conducted between 2011 and 2015, at baseline and roughly every 12 months thereafter. Young women were in different grades at enrollment and could have had fewer than 4 visits if they were expected to graduate before the end of the study period. An additional HIV and HSV2 test was conducted for some girls around the time of expected graduation from high school or when the study was completed to capture more person time in the study if eligibility was met (termed the graduation test). This test was typically around 6 months after the previous annual visit.

\section{Exposure, Outcome, and Mediator Ascertainment}

The exposure of school attendance was constructed using school attendance registers collected directly from high schools. School attendance was defined as the average percentage of days attended in the months of February, May, and August between surveys, as these months were most representative of normal attendance because of the absence of holidays or examinations. School attendance was dichotomized as high ( $\geq 80 \%$ of school days) versus low ( $<80 \%$ of school days) attendance as per the original cash transfer study. ${ }^{26}$

The mediator of "having an older partner" was defined as having had at least 1 sexual or nonsexual partner 5 or more years older at each follow-up visit. Partners with whom there was no reported sexual relationship were included to account for potential misreporting about sexual behaviors. The mediator of the "number of sexual partners" was defined as having zero, 1 or $>1$ sex partners in the past 12 months at each visit. The outcomes of incident HIV and HSV-2 infection were defined as new cases after the first follow-up visit, as described by testing procedures in the main article. ${ }^{26}$ We include incident cases from the second visit onward to ensure that all infections occurred after the mediator and exposure ascertainment.

\section{Statistical Analysis}

We used the potential outcomes framework to define the total effect as the risk difference (RD) comparing the 4year cumulative incidence of HIV or HSV-2 had all young women had high attendance to the 4-year cumulative incidence of HIV or HSV-2 had all young women had low attendance. Likewise, the controlled direct effect (CDE) was defined as the RD (high versus low attendance) that would have been observed if attendance was prevented from affecting each of the mediators. ${ }^{27,28}$ Specifically, our study explored if partner age difference or the number of partners were mediators in the relationship between school attendance and incident $\mathrm{HIV} / \mathrm{HSV}-2$ infection by estimating the CDE of 

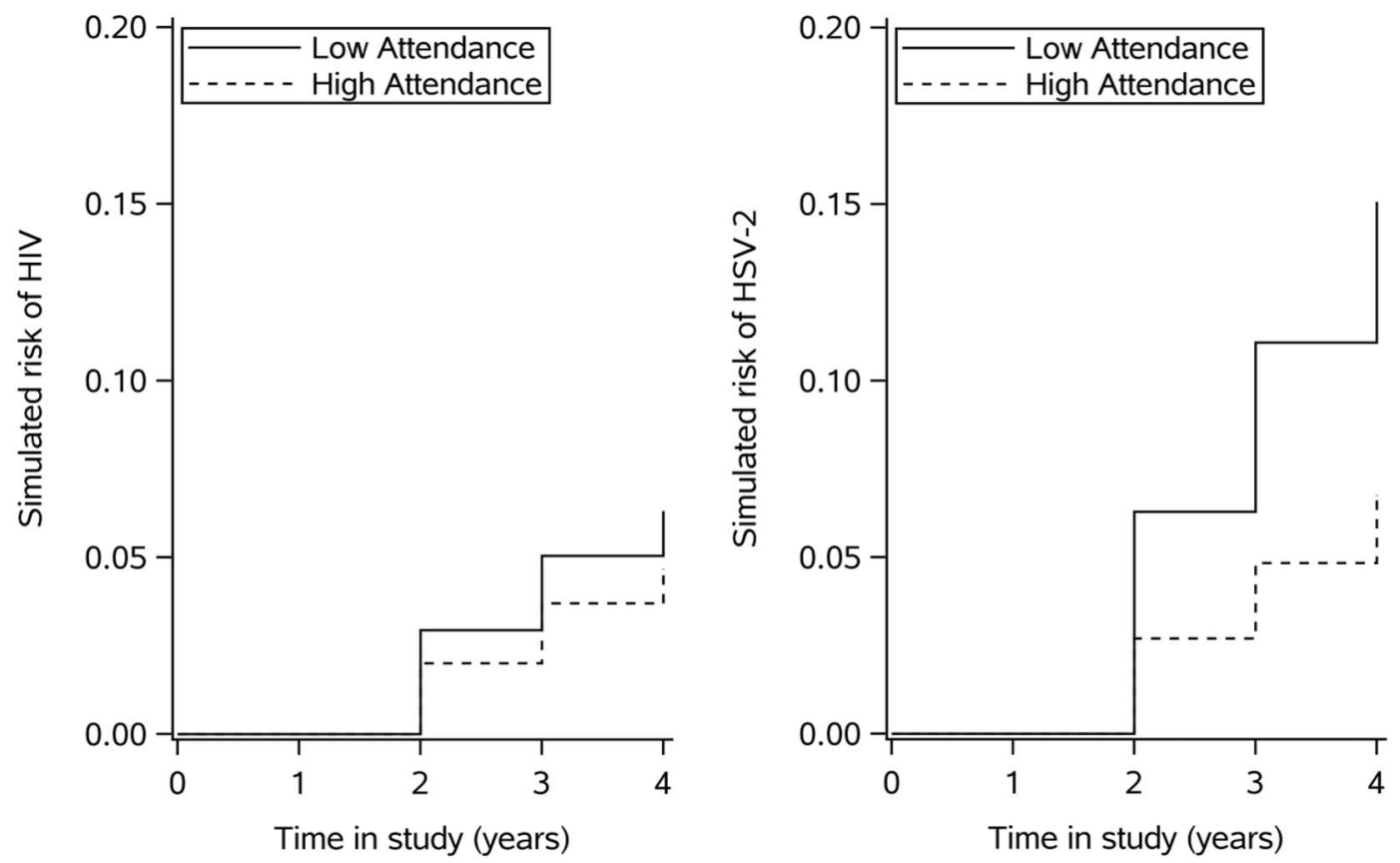

FIGURE 2. Cumulative incidence of HIV and HSV-2 by time since study enrollment and attendance in a Monte Carlo sample of 10,000 , accounting for confounding.

attendance while intervening to fix (ie, control for) the mediators. ${ }^{29-31}$ In our study, the CDE can be interpreted as the effect of school attendance on HIV and HSV-2 that occurs through mechanisms other than by reducing partner age and number. We chose to estimate the CDE for mediation rather than the natural direct or indirect effect because it does not require as many assumptions including the assumption that there is no mediator-outcome confounder that is affected by the exposure, which we cannot assume in this analysis. ${ }^{32}$ Additionally, we examined the CDE under several different scenarios or "interventions" including (1) prevent young women from having any sexual partners and older sexual or nonsexual partners; (2) set women to have 1 sexual partner and prevent women from having any older sexual or nonsexual partners; (3) reduce the number of women with an older partner by $50 \%$; (4) set young women to have fewer partners (those with $\geq 2$ have 1 partner and those with 1 have 0 partners); and (5) set young women to have fewer partners and reduced the number of women with an older partner by $50 \%$.

We estimated the total effect and CDE using the parametric g-formula, a generalization of standardization that allows us to account for time-varying confounding and accommodate interactions between school attendance and

TABLE 2. CDE of School Attendance on Incident HIV and HSV-2 by Different Levels of the Mediators Partner Number and Partner Age Difference

\begin{tabular}{|c|c|c|c|c|c|c|}
\hline & \multicolumn{3}{|c|}{ HIV } & \multicolumn{3}{|c|}{ HSV-2 } \\
\hline & Risk (\%) & RD\% (95\% CI) & RR $(95 \%$ CI $)$ & Risk (\%) & RD\% (95\% CI) & RR $(95 \%$ CI $)$ \\
\hline \multicolumn{7}{|l|}{ Total effect } \\
\hline High attendance & 4.7 & $-1.6(-2.3$ to -1.0$)$ & $0.74(0.66$ to 0.83$)$ & 6.7 & $-8.3(-9.1$ to -7.5$)$ & $0.45(0.41$ to 0.49$)$ \\
\hline Low attendance & 6.3 & 0 & 1.0 & 15.1 & 0 & 1 \\
\hline \multicolumn{7}{|c|}{ CDE: no older partners } \\
\hline High attendance & 4.6 & $-1.2(-1.8$ to -0.7$)$ & $0.79(0.70$ to 0.88$)$ & 6.3 & $-6.2(-7.1$ to -5.4$)$ & $0.51(0.46$ to 0.55$)$ \\
\hline \multicolumn{7}{|c|}{ CDE: no sexual partners } \\
\hline High attendance & 3.2 & $-0.4(-0.9$ to 0.1$)$ & $0.89(0.77$ to 1.03$)$ & 5.8 & $-6.9(-7.8$ to -6.1$)$ & $0.46(0.41$ to 0.50$)$ \\
\hline Low attendance & 3.6 & 0 & 1 & 12.8 & 0 & 1 \\
\hline \multicolumn{7}{|c|}{ CDE: 1 sexual partner } \\
\hline High attendance & 7.0 & $-0.8(-1.6$ to -0.1$)$ & $0.89(0.81$ to 0.98$)$ & 8.2 & $-7.6(-8.5$ to -6.7$)$ & $0.52(0.48$ to 0.56$)$ \\
\hline Low attendance & 7.8 & 0 & 1 & 15.8 & 0 & 1 \\
\hline
\end{tabular}

$\mathrm{RD}$, risk difference, $\mathrm{RR}$, risk ratio. 
the mediators. ${ }^{27,31,32}$ Details on using the parametric gformula for mediation analysis have been described previously. ${ }^{27,33-37}$ Briefly, we created a simulated version of our data set with no confounding in which we set variables to mimic interventions (see technical appendix for more detail) on school attendance and each mediator.

Confounders were selected using a directed acyclic graph for the relationship between school attendance and HIV and for the relationship between school attendance and HSV-2. We included the exposure-outcome confounders of time, age at baseline, intervention assignment at baseline, SES at baseline (defined using quartiles of household assets), time-varying orphan status, time-varying alcohol use, time-varying children's depression inventory score,${ }^{38,39}$ and time-varying revised children's manifest anxiety score. ${ }^{40}$ In addition, we included HSV-2 status in the model for the outcome of HIV. We also included the mediator-outcome confounders of depression, anxiety, and alcohol use in all models (all time varying). An interaction term between the mediators, partner age difference and partner number, was also included in the model, but we were unable to include an interaction term between exposure and mediators because of sparse data (see technical appendix). We examined SES defined using both household assets and parental educational attainment but ultimately chose assets because results were similar and assets had less missing data.

Risk of HIV and HSV-2 for each exposure pattern (the combination of interventions on exposure and mediators) was estimated using the complement of the Kaplan-Meier estimator extended to account for time-varying exposures. ${ }^{33} \mathrm{We}$ compared the risk of HIV and HSV-2 at the end of the study period (4 years to account for the extra graduation test) under each exposure plan using RDs and risk ratios. Ninety five percent confidence intervals (CI) were computed using the standard errors from 200 nonparametric bootstrap resamples. SAS version 9.3 (SAS Institute, Inc., Cary, NC) was used for all analyses.

\section{RESULTS}

A total of 2086 young women who were HIV negative at baseline and at the first follow-up, and had at least 2 follow-up visits following baseline were included in our HIV cohort. For the HSV-2 cohort, we further excluded prevalent cases of HSV-2 at baseline and those who were missing HSV-2 status for a total of 1963 young women with 4192 visits over the study period. In the observed data, there were 74 incident HIV infections and 117 incident HSV-2 infections from follow-up visit 2 to the end of the study period. Of the 4450 visits with school attendance data, girls who were HIV negative at baseline had high attendance during $95.2 \%$ of visits $(n=4234)$. At baseline in the observed data, $5.1 \%(\mathrm{n}=105)$ had a partner 5 or more years older, $78.9 \%$ had zero partners $(n=1626)$, and $16.7 \%(n=344)$ had 1 partner in the past 12 months (Table 1 ).

The risk of HIV at 4 years of follow-up time would be $6.3 \%$ if all young women had low school attendance and $4.7 \%$ if all young women had high attendance over the entire study period (Fig. 2 and Table 2). Cumulative incidence of HIV and HSV-2 estimated under no intervention on exposure or mediators (the "natural course") was similar to the cumulative incidence of the outcomes in the observed data (HIV 4.9\%; HSV-2 8.1\%; Appendix Figure 1, http://links. lww.com/QAI/B180). Table 2 shows the total effect of school attendance compared with the CDE of attendance when young women also do not have an older partner, when they have 1 partner and zero partners. The estimated RD at 4 years for the effect of high versus low school attendance on HIV was $-1.6 \%(95 \% \mathrm{CI}:-2.3 \%$ to $-1.0 \%)$. The RD of $-1.6 \%$ represents the total effect of school attendance on HIV operating through all pathways, including partner age and number. When we removed the effect of attendance on partner age by setting all girls to have a younger partner, the $\mathrm{RD}$ for the effect of school attendance on HIV was $-1.2 \%$

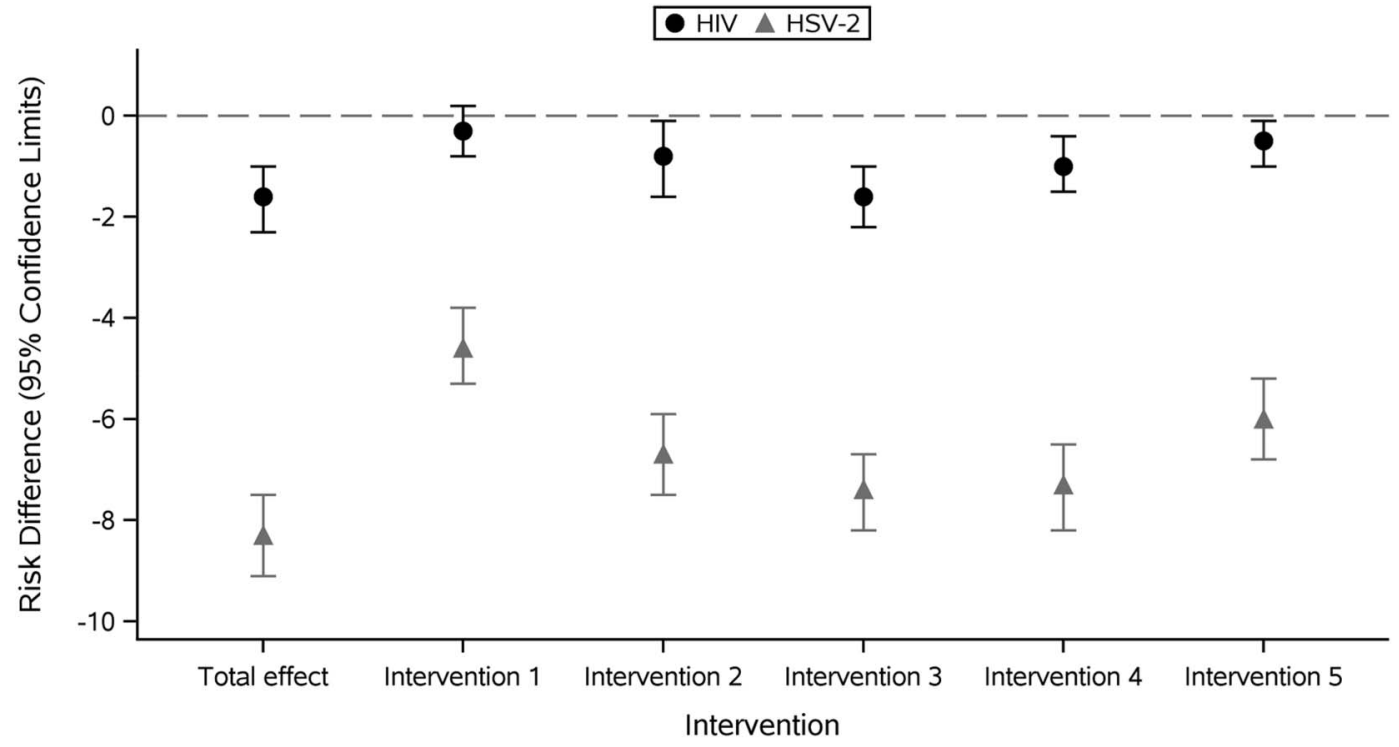

FIGURE 3. CDE of school attendance on incident HIV and HSV-2 with various interventions on the mediators partner age difference and partner number. 
(95\% CI: $-1.8 \%$ to $-0.7 \%$ ). When we removed the effect of school attendance on partner number by setting young women have 0 partners, the RD for the effect of school attendance on HIV was $-0.4 \%$ (95\% CI: $-0.9 \%$ to $0.1 \%)$. When we set the number of partners to 1 partner (even those with 0 partners), the $\mathrm{RD}$ at 4 years was $-0.8 \%$ ( $95 \% \mathrm{CI}-1.6 \%$ to $-0.1 \%)$.

The risk of HSV-2 at 4 years was $15.1 \%$ if all young women had low attendance and $6.7 \%$ if all young women had high attendance over the entire study period (Fig. 2 and Table 2 ). The estimated RD for the total effect of high versus low school attendance on HSV-2 was higher than that on HIV at $-8.3 \%$ (95\% CI: $-9.1 \%$ to $-7.5 \%$ ) (Table 2). When we removed the effect of attendance on partner age, the RD at 4 years was $-6.2 \%(95 \% \mathrm{CI}:-7.1 \%$ to $-5.4 \%)$ When all young women had 0 partners, the RD was $-6.9 \%$ (95\% CI: $-7.8 \%$ to $-6.1 \%$ ). When we set the number of partners to 1 , the RD at 4 years was $-7.6 \%$ (95\% CI: $-8.5 \%$ to $-6.7 \%$ ).

Figure 3 shows the effect of school attendance on HIV and HSV-2 under 5 potential scenarios where we have jointly removed the effect of school attendance on both partner age difference and partner number. For incident HIV, the RD for the $\mathrm{CDE}$ of school attendance was attenuated from the total effect in all scenarios. Under intervention 1, the RD was almost zero (RD, $-0.3 \%$; $95 \%$ CI: $-0.8 \%$ to $0.2 \%$ ). Even under intervention 5 where we do not completely set behaviors, the effect of school attendance on HIV acquisition was almost removed (RD, $-0.5 \% ; 95 \%$ CI: $-1.0 \%$ to $-0.1 \%$ ). For incident HSV-2 infection, the RD for the effect of school attendance on HSV-2 was again attenuated from the total effect in all scenarios, although the effect was never completely removed. The RD for the effect of school attendance on HSV-2 was closest to the null under intervention 1 when we intervened to set all young women to have younger partners and zero partners (RD, $-4.6 \%$; $95 \%$ CI $-5.3 \%$ to $-3.8 \%$ ).

\section{DISCUSSION}

In this cohort of AGYW in rural South Africa, we found that partner age and partner number mediated the relationship between school attendance and HIV and HSV-2 acquisition. School attendance was associated with the risk of both incident HIV and HSV-2. The effect of school attendance on incident infection was nearly removed when also intervening on the number of older partners and partner number, suggesting that a large proportion of protective effect of school attendance on HIV acquisition in this cohort is the result of sexual partner characteristics. In fact, the effect of school attendance on HIV, although primarily driven by partner number, is null after accounting for partner selection.

Our results are compatible with the theory that young women who attend more school are at the lower risk of HIV and HSV-2 infection because of their sexual network structure. ${ }^{7,16}$ Previous studies have reported a lower prevalence of HIV and HSV-2 in young men and women attending school and associations between school attendance, partner age difference, and number of partners. ${ }^{7,16,41} \mathrm{We}$ found that school attendance was associated with a reduced risk of HIV and HSV-2, and we add to the literature by formally assessing mediation to illustrate that partner age difference and partner number are mediators of these relationships. Although it may be impossible to do things like "prevent older partners" explicitly, these results are important because they show illustratively that school attendance naturally prevents HIV and HSV-2 by providing periods of structure and supervision in young adults' lives, which reduce opportunities for sexual activity and promote safer networks. $3,16,19$

The results for mediation of the effect of school attendance on HIV differed slightly from the results for HSV2. Partner selection can almost entirely explain the relationship between school attendance and HIV. However, removing the effect of school attendance on partner age and partner number removed only some of the effect of school attendance on HSV2. These results indicate that there are likely other mechanisms that are additional mediators of the relationship between school attendance and HSV-2. For example, because HSV-2 is more transmissible than HIV, condom use to reduce efficiency of transmission per contact might be an important determinant of infection that is linked to schooling. ${ }^{42,43}$ It is also possible, given the higher prevalence of HSV-2 in general, that younger men are more likely to have HSV-2, making network patterns different than HIV. ${ }^{44}$

It should be noted that information on sexual behaviors was self-reported, and there may have been some misreporting in the study despite the use of audio computer-assisted self-interview to minimize reporting bias. ${ }^{25}$ Misreporting about sexual behaviors is apparent in the remaining risk of HIV/HSV-2 infection after we fix all young women to have zero partners (we would expect that girls would not get infected if they do not have sex). We do show that even if young women had 1 partner overall or if they had $50 \%$ fewer older partners and fewer partners overall, the effect of school attendance on the incidence of infection would still be much smaller. A sensitivity analysis was done setting all HIV cases in girls with 0 partners to have 1 partner and to have 2 partners (Appendix Table 2 and Table 3, http://links.lww. com/QAI/B180) and we found similar results. However, the total effect and the mediation by partner number were larger, and mediation by partner age difference was not as pronounced. It is likely that girls who misreport sexual activity would also misreport partner age, as they may not have included the ages of any partners on the survey. Moreover, when we assumed that girls who were HIV positive and had 0 partners also had older partners, we found again similar results and mediation by partner age difference (Appendix Table 3, http://links.lww.com/QAI/B180).

In addition, the assumption of no unmeasured confounding is a strong assumption that is impossible to assess in the data. ${ }^{31}$ It is possible that there are confounders we did not measure or include in our models. However, we did explore measured confounding by examining the effect of adding and removing different variables to our models. We also used the $\mathrm{CDE}$ instead of the indirect and natural direct effects for mediation to avoid the assumption of no mediator-outcome confounders affected by prior exposure. Second, the parametric $\mathrm{g}$-formula assumes that the parametric models used to predict our variables are correctly specified. This assumption is not testable, but covariate distributions and cumulative incidence functions in the predicted natural course were a close fit to the 
observed data, suggesting that the models were adequately specified. $^{33}$ Finally, one benefit of using causal inference methods for mediation is to include exposure-mediator interactions; however, we were unable to do so in our analysis because of sparse data (see technical appendix). Therefore, our analysis assumes that the effects of exposure and mediators are multiplicative.

It should be noted that the data are from a randomized controlled trial where all young women were already enrolled in school at study enrollment. Previous analyses of the data have shown potential selection bias where young women in the study may have been more likely to attend school than the underlying population and a Hawthorne effect where young women in the trial were less likely to drop out of school because of trial participation. ${ }^{45}$ However, we would expect the relationship between schooling and HIV/HSV-2 to remain similar.

We found that school attendance was associated with both incident HIV and HSV-2 infection and are the first to show that partner age difference and the number of partners mediate the relationships between school attendance and incident HIV and HSV-2 infection. These findings are significant as education has been one of the factors that is most consistently associated with preventing HIV in AGYW and we provide a better understanding about how that

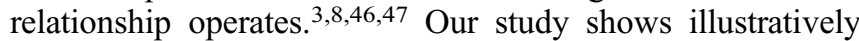
that school attendance naturally reduces the risk HIV and HSV-2 acquisition among AGYW by providing periods of structure and supervision in young adults' lives, which encourage younger and fewer partners. Changes in these partnership factors will ultimately reduce opportunities for sexual activity and promote safer networks where girls are not exposed to infection. Interventions to prevent infections in young women should focus on keeping girls in school and creating other environments like school that constructively occupy time and provide a safe space where young women can associate with their peers. Alternatively, given the importance of partner age and number to adolescent HIV acquisition, efforts to provide biomedical interventions like Pre-Exposure Prophylaxis to girls who are sexually active, out of school, or who have older partners may help to prevent new infections. Schooling is one of the strong preventative interventions for HIV and HSV-2 among AGYW. Evidence that this effect operates through partner selection should encourage interventions to keep girls in school and the development of interventions that emulate this supervised and structured environment, potentially changing sexual networks and norms.

\section{ACKNOWLEDGMENTS}

The authors thank the HPTN 068 study team and all trial participants.

\section{REFERENCES}

1. Gómez-Olivé FX, Angotti N, Houle B, et al. Prevalence of HIV among those 15 and older in rural South Africa. AIDS Care. 2013;25: $1122-1128$.
2. Jewkes R, Wood K, Duvvury N. "I woke up after i joined Stepping Stones": meanings of an HIV behavioural intervention in rural South African young people's lives. Health Educ Res. 2010;25:1074-1084.

3. Jukes M, Simmons S, Bundy D. Education and vulnerability: the role of schools in protecting young women and girls from HIV in southern Africa. AIDS. 2008;22(suppl 4):S41-S56.

4 Pettifor A, Bekker L-G, Hosek S, et al. Preventing HIV among young people: research priorities for the future. J Acquir Immune Defic Syndr. 2013; 63(suppl 2):S155-S160.

5. Hardee K, Gay J, Croce-Galis M, et al. What HIV programs work for adolescent girls? J Acquir Immune Defic Syndr. 2014; 66(suppl 2):S176S185.

6. Gavin L, Galavotti C, Dube H, et al. Factors associated with HIV infection in adolescent females in Zimbabwe. J Adolesc Health. 2006;39: 596.e11-e18.

7. Hargreaves JR, Morison La, Kim JC, et al. The association between school attendance, HIV infection and sexual behaviour among young people in rural South Africa. J Epidemiol Community Health. 2008;62: 113-119.

8. Pettifor AE, Levandowski BA, Macphail C, et al. Keep them in school: the importance of education as a protective factor against HIV infection among young South African women. Int J Epidemiol. 2008;37:12661273.

9. Baird S, Chirwa E, McIntosh C, et al. The short-term impacts of a schooling conditional cash transfer program on the sexual behavior of young women. Health Econ. 2010;19(suppl):55-68.

10. Birdthistle I, Floyd S, Nyagadza A, et al. Is education the link between orphanhood and HIV/HSV-2 risk among female adolescents in urban Zimbabwe? Soc Sci Med. 2009;68:1810-1818.

11 Stoner M, Pettifor A, Edwards J, et al. The effect of school attendance and school dropout on incident HIV and HSV-2 among young women in rural South Africa enrolled in HPTN 068. AIDS. 2017;31:2127-2134.

12. Boerma JT, Weir SS. Integrating demographic and epidemiological approaches to research on HIV/AIDS: the proximate-determinants framework. J Infect Dis. 2005;191(suppl):S61-S67.

13. Zambuko O, Mturi AJ. Sexual risk behaviour among the youth in the era of HIV/AIDS in South Africa. J Biosoc Sci. 2005;37:569-584.

14. Zuilkowski SS, Jukes MC. The impact of education on sexual behavior in sub-Saharan Africa: a review of the evidence. AIDS Care. 2012;24: $562-576$

15. Harrison A, Cleland J, Frohlich J. Young People's sexual partnerships in KwaZulu-Natal, South Africa: patterns, contextual influences, and HIV risk. Stud Fam Plann. 2008;39:295-308.

16. Stoner MCD, Edwards JK, Miller WC, et al. Effect of schooling on agedisparate relationships and number of sexual partners among young women in rural South Africa enrolled in HPTN 068. J Acquir Immune Defic Syndr. 2017;76:e107-e114.

17. Kretzschmar M, Morris M. Measures of concurrency in networks and the spread of infectious disease. Math Biosci. 1996;133:165-195.

18. Morris M. Concurrent partnerships and syphilis persistence: new thoughts on an old puzzle. Sex Transm Dis. 2001;28:504-507.

19. Barnes GM, Hoffman JH, Welte JW, et al. Adolescents' time use: effects on substance use, delinquency and sexual activity. $J$ Youth Adolesc. 2007;36:697-710

20. Osgood DW, Wilson JK, O'Malley PM, et al. Routine activities and individual deviant behavior. Am Sociol Rev. 1996;61:635.

21. Hawdon JE. Daily routines and crime: using routine activities as measures of Hirschi's involvement. Youth Soc. 1999;30:395-415.

22 Hirschi T. A Control Theory of Delinquency; 1969. Available at: http:// ovidsp.ovid.com/ovidweb.cgi? $\mathrm{T}=\mathrm{JS} \& \mathrm{PAGE}=$ reference $\& \mathrm{D}=\mathrm{psyc} 6 \& \mathrm{NEWS}=\mathrm{N} \& A \mathrm{~N}=2007-05262-005$.

23. Burkett SR, White M. Hellfire delinquency another look. J Sci Study Relig. 1974;13:455-462.

24. Cohen LE, Felson M. Social change and rate crime trends: A routine activity approach. Am Sociol Rev. 1979;44:588

25 Pettifor A, MacPhail C, Selin A, et al. HPTN 068: A randomized control trial of a conditional cash transfer to reduce HIV infection in young women in South Africa-Study design and baseline results. AIDS Behav. 2016;20:1863-1882.

26 Pettifor A, MacPhail C, Hughes JP, et al. The effect of a conditional cash transfer on HIV incidence in young women in rural South Africa (HPTN 
068): a phase 3 randomised controlled trial. Lancet Glob Health. 2016;12: e978-e988

27. Lin S-H, Young J, Logan R, et al. Parametric mediational g-formula approach to mediation analysis with time-varying exposures, mediators, and confounders. Epidemiology. 2016;28:1.

28. Westreich D, Cole SR, Young JG, et al. The parametric g-formula to estimate the effect of highly active antiretroviral therapy on incident AIDS or death. Stat Med. 2012;31:2000-2009.

29. VanderWeele TJ. Causal mediation analysis with survival data. Epidemiology. 2012;22:582-585.

30. VanderWeele TJ. Marginal structural models for the estimation of direct and indirect effects. Epidemiology. 2009;20:18-26.

31. Mumford SL, Schisterman EF, Siega-Riz AM, et al. Effect of dietary fiber intake on lipoprotein cholesterol levels independent of estradiol in healthy premenopausal women. Am J Epidemiol. 2011;173:145-156.

32. Naimi AI, Kaufman JS, MacLehose RF. Mediation misgivings: ambiguous clinical and public health interpretations of natural direct and indirect effects. Int J Epidemiol. 2014;43:1656-1661.

33. Edwards JK, McGrath LJ, Buckley JP, et al. Occupational radon exposure and lung cancer mortality: estimating intervention effects using the parametric g-formula. Epidemiology. 2014;25:829-834.

34. Naimi A, Cole S, Hudgens M, et al. Estimating the effect of cumulative occupational asbestos exposure on time to lung cancer mortality: using structural nested failure-time models to account for healthy-worker survivor bias. Epidemiology. 2014;25:246-254.

35. Danaei G, Pan A, Hu FB. Hernán M a. Hypothetical midlife interventions in women and risk of type 2 diabetes. Epidemiology. 2013;24:122-128.

36. Lajous M, Willett WC, Robins J, et al. Changes in fish consumption in midlife and the risk of coronary heart disease in men and women. $\mathrm{Am} J$ Epidemiol. 2013;178:382-391.
37. Vanderweele TJ, Tchetgen Tchetgen E. Mediation analysis with timevarying exposures and mediators. J R Stat Soc Series B Stat Methodol. 2014; $168: 1-22$.

38. Kovacs M. The children's depression inventory (CDI). Psychopharmacol Bull. 1985;21:995-998.

39. Cluver L, Gardner F, Operario D. Psychological distress amongst AIDSorphaned children in urban South Africa. J Child Psychol Psychiatry. 2007;48:755-763.

40. Boyes ME, Cluver LD. Performance of the revised Children's manifest anxiety scale in a sample of children and adolescents from poor urban communities in cape Town. Eur J Psychol Assess. 2013;29:113-120.

41. Baird SJ, Garfein RS, McIntosh CT, et al. Effect of a cash transfer programme for schooling on prevalence of HIV and herpes simplex type 2 in Malawi: a cluster randomised trial. Lancet. 2012;379:1320-1329.

42. Wald A, Langenberg AGM, Link K, et al. Effect of condoms on reducing the transmission of herpes simplex virus type 2 from men to women. JAMA J Am Med Assoc. 2001;285:3100-3106.

43. Wald A, Krantz E, Selke S, et al. Knowledge of partners' genital herpes protects against herpes simplex virus type 2 acquisition. $J$ Infect Dis. 2006;194:42-52.

44. Jewkes R, Nduna M, Levin J, et al. Impact of Stepping Stones on incidence of HIV and HSV-2 and sexual behaviour in rural South Africa: cluster randomised controlled trial. BMJ. 2008;337:a506.

45. Rosenberg M, Pettifor A, Twine R, et al. Evidence for sample selection effect and Hawthorne effect in behavioural HIV prevention trial among young women in a rural South African community. BMJ Open. 2018;8:e19167.

46 Hardee K, Gay J, Croce-Galis M. What HIV programs work for adolescent girls? J Acquir Immune Defic Syndr. 2014;66(suppl 2): S176-S185.

47. Acevedo-Polakovich I. Youth Go: An Out-of-School Time Program Friendly Approach to Gathering Youth Perspectives. 2017. In Press. 